\title{
What is nature capable of? Evidence, ontology and speculative medical humanities
}

\author{
Martin Savransky, Marsha Rosengarten
}

Department of Sociology, Goldsmiths, University of London, London, UK

\section{Correspondence to} Dr Martin Savransky, Department of Sociology, Goldsmiths, University of London, London SE14 JW, UK; m.savransky@gold.ac.uk

Accepted 25 April 2016 Published Online First 24 May 2016

CrossMark

To cite: Savransky M, Rosengarten M. Med Humanit 2016;42:166-172.

\section{ABSTRACT}

Expanding on the recent call for a 'critical medical humanities' to intervene in questions of the ontology of health, this article develops a what we call a 'speculative' orientation to such interventions in relation to some of the ontological commitments on which contemporary biomedical cultures rest. We argue that crucial to this task is an approach to ontology that treats it not as a question of first principles, but as a matter of the consequences of the images of nature that contemporary biomedical research practices espouse when they make claims to evidence, as well as the possible consequences of imagining different worlds in which health and disease processes partake. By attending to the implicit ontological assumptions involved in the method par excellence of biomedical research, namely the randomised controlled trial (RCT), we argue that the mechanistic ontology that tacitly informs evidence-based biomedical research simultaneously authorises a series of problematic consequences for understanding and intervening practically in the concrete realities of health. As a response, we develop an alternative ontological proposition that regards processes of health and disease as always situated achievements. We show that, without disqualifying RCT-based evidence, such a situated ontology enables one to resist the reduction of the realities of health and disease to biomedicine's current forms of explanation. In so doing, we call for medical humanities scholars to actively engage in the speculative question of what nature may be capable of.

\section{INTRODUCTION: CRITICAL MEDICAL HUMANITIES, ONTOLOGY AND SPECULATION}

In the introduction to a recent special issue on the theme of 'critical medical humanities', William Viney and his collaborators call for scholars to rethink the possible roles of the medical humanities in its entanglements with biomedical cultures. ${ }^{1}$ In this programmatic text, they pose a question for a future medical humanities that could act as a tool for addressing not just the meanings and contexts of health, disease and illness, but 'their very production, concrescence, and dispersal', a question that we seek to take up and expand on throughout this article: 'Can the medical humanities intervene more explicitly in ontological questions [...] rather than, as has commonly been the case, leaving such questions largely to the domains of the life sciences and biomedicine? ${ }^{1}$

The importance of reclaiming questions of ontology in biomedical cultures and practices is paramount. Not only because these questions have been historically relegated to the life sciences and biomedicine, but because, in the hands of a contemporary biomedical culture by and large dominated by the so-called paradigm of evidencebased medicine (EBM), questions of ontology-of the complex and heterogeneous natures of the worlds in which health and disease emerge-have been deemed dated, superseded by a data-intensive, correlational approach to medical evidence. ${ }^{2-4}$ As we show, this move within EBM has the pernicious effect of letting certain research methodologies prescribe in advance what is and what is not relevant to understanding and intervening in processes of health and disease everywhere and always, despite the not infrequent difficulties such directives encounter in becoming effective in concrete situations.

Because to interrogate the ontologies of health in relation to biomedical practices is to explore the kind of realities of health that these practices enable to come into existence, as well as those that they prevent from coming into existence, the call for medical humanities to intervene in ontological questions simultaneously involves an invitation to actively participate in the problem-at once philosophical and ethical, scientific and political-of what counts as evidence and of what image of the world certain methods for producing and understanding evidence in (bio)medicine espouse. We take it, moreover, as an invitation to imaginatively disclose possibilities for alternative configurations of the worlds in which the realities of health and disease are produced. The aim of this paper is thus to develop such an understanding as a possible orientation to the question of how to intervene in ontological dimensions of health-an orientation we associate with the idea of a 'speculative medical humanities'.

Such a task must, above all, abandon the conventional view that insofar as ontology designates the question of 'being', of what does and may 'exist', it must be regarded as belonging to an order of inquiry that is more fundamental than, and therefore independent of, practical questions of ethics and politics. Instead of treating ontology as a matter of principle, what is at stake, we suggest, is the possibility of developing an ontological intervention as a pragmatic art of consequences. ${ }^{5}$ An art, first, of witnessing the effects, both welcome and dangerous, of the images of nature (and culture) that contemporary biomedical research practices tacitly espouse when they make claims to evidence. And second, of speculating on the possible consequences of imagining alternative images in which the realities of health and disease can prompt novel understandings, where the evidence produced by biomedical methods might acquire different meanings, and where other forms of knowledge, of evidence and of ignorance may be integrated. ${ }^{6}$ 
Intervening in ontological questions of health and disease through an art of consequences is therefore neither about giving a plausible account of the ultimate reality of health and disease, nor about attempting to determine what is 'natural' and what is 'cultural' in relation to given conditions, or whether or not health conditions are real. ${ }^{7}$ Rather, it is an attempt to approach medical realities with the always open, exploratory question: 'what is nature capable of?' It is, in other words, a speculative exercise in transforming the horizon of possibilities of the field in which a problem is posed-a thought experiment concerned, as philosopher of science Isabelle Stengers puts it, ${ }^{8}$ with 'creating possibles, that is [with] making visible the directives, evidences, and rejections that those possibles must question before they themselves can become perceptible'. Unlike many philosophies of science and medicine that have been concerned with legislating in the abstract the so-called rational requirements of a modern science that could finally legitimise itself as the only objective interpreter of nature, the task of creating possibles is animated by a different, and more risky, problem. It involves paying attention to, and honouring, the situated adventures of scientific, medical and other practitioners, ${ }^{8-13}$ while resisting the temptation to submit one's questions to the acquired authority of the tradition and institution known as modern science. The authority, that is, to construct 'a perfectly convincing argument that, as if by chance, has the capacity to dissimulate or condemn a question it doesn't feel very certain about'. 1415

Making those condemned questions resonate against the directives and rejections that have dissimulated their importance is central to the task of creating possibles. And questioning such directives and rejections often involves, as is the case in this article, a close exploration of the procedures by which certain forms of thinking, knowing and doing operate. That is to say, it involves an exploration of their guiding concepts and reasons, as well as their methodologies. ${ }^{16}$ When developed as an art of consequences, however, such questioning is never premised on the gesture of debunking the internal inconsistencies of a method for its own sake. ${ }^{17}$ Rather, it is oriented by the attempt to disclose the ontological commitments certain methods hold and to experience a mutation of their consequences as the possibility of an alternative is imaginatively developed.

Here we engage in such a form of experimentation by interrogating the ontological commitments involved in one crucial aspect on which the production of biomedical evidence rests. That is, the fundamental distinction between, on the one hand, the concept and measure of 'efficacy', or the testing of the effects of a biomedical intervention under so-called 'optimal' conditions; and on the other, the correlative concept of 'effectiveness' or the assessment of an 'efficacious' intervention under so-called 'real world' conditions. We argue that this split, foundational as it is for the 'evidence' generated by the method of the randomised controlled trial (RCT) as well as for the policy and clinical decisions that follow from it, simultaneously separates nature-and biology-from contingencies deemed irrelevant to them and discloses an image of a natural world composed of bits of matter distributed probabilistically across bodies, with high degrees of regularity. This is an image that not only divides natures and cultures-situating the reality of health on the former side of the divide only-but also enables the 'nature' of health processes to rest on a mechanistic ontology that, while it is the basis on which proponents of EBM raise the RCT to the status of a gold standard, it is also the source of countless problematic consequences in practice.
Drawing inspiration from a diverse range of thinkers-philosophers, biologists and medical humanities scholars—6 8 8 $18-26$ who, in diverse ways, have been concerned with making resonate the question of what nature may be capable of, we engage in a speculative exercise of proposing an image of nature that does not reject the knowledge-practices of biomedical research but enables one to rethink the manner in which their evidentiary claims take hold. This, we suggest, can be accomplished by re-situating medical realities in a world of dynamic and connected situations, both natural and cultural, which are governed neither by mechanistic forms of order nor by utter chaos, but rather by always partial, incomplete and dynamic forms of organisation, novelty and change.

\section{ON THE HEALTH OF MECHANISMS: THE BIFURCATED NATURE OF BIOMEDICAL CULTURE}

As is well known, leading proponents of EBM define it as 'the conscientious, explicit and judicious use of current best evidence in making decisions about the care of individual patients'. ${ }^{27}$ While such a definition seems irreproachable-what would one recommend care to be based on if not the 'best' evidence-the central question becomes, of course, what counts as 'best' evidence and why. The literature on EBM rapidly makes apparent that 'Randomised controlled trials [...] and systematic reviews are the most reliable methods of determining the effects of treatment'. ${ }^{28}$ Not only does the RCT occupy the highest position within EBM's hierarchies of evidence, but it has been deemed the 'gold standard' of scientific evidence for medical treatments-and indeed for other kinds of interventions as well. ${ }^{26}$ The metaphoric name itself suggests that it is deemed not just the 'best' kind of evidence, but the norm against which any form of evidence is to be compared and judged. ${ }^{2} 29$ So what does 'evidence' mean in the context of RCTs?

Once the initial phases of safety testing are carried out, RCTs are deployed to test the efficacy of an intervention, that is, the ability of a treatment to produce benefit if applied ideally under experimentally controlled, 'optimal' conditions. In order to achieve this measure, RCTs work by a method of difference, inherited from the philosophy of John Stuart Mill, ${ }^{30}$ that seeks to evaluate the differential values of one variable by staging a comparison between two or more groups that are otherwise deemed equivalent or homogeneous. At stake in the institution of such ideal conditions is the attempt, on the negative side, to rationally cancel out, by way of techniques of randomisation, ${ }^{2}$ every other co-intervening factor in the identification of a coming about of an effect. On the positive side, the attempt is to produce evidence that the single cause in question is responsible for the effect of a treatment.

If and when the efficacy of an intervention is established, further studies may be implemented in which the said intervention is tested under so-called 'real world' conditions, thereby loosening the methodological requirements of the trial with the aim of assessing the effects of an-efficacious-intervention in the context of more complex, diverse, social, economic, infrastructural and cultural circumstances of intervention. It is this latter test that receives the name of 'effectiveness'. ${ }^{28}$

For those engaged in social studies of medical trials, ${ }^{31} 32$ in practice such distinctions are never as clear-cut as adherence to a protocol would suggest. On the one hand, measuring efficacy often requires practical adjustments and adaptations that, while perhaps not always reported, situate the trial in relation to those on whom the tests are conducted and force its procedures to adjust in relation to the course of events that constitute it. On the other, once efficacy measures have been established and 
have informed the making of standards of medical care, the practical application of those standards always demands, again, a mutual adjustment between the demands of the standard and the demands of the clinical situation at stake. ${ }^{29} 33$

Aware that the distinctions between 'efficacy' and 'effectiveness' are practically problematic, epidemiologists, for their part, have attempted to develop classificatory schemes that present the relationship between the two concepts as a continuum, while salvaging their epistemological and ontological differences. ${ }^{34}$ Thus, from the outset, these epidemiologists tellingly associate 'efficacy' with the task of providing an 'explanation', that is, of 'testing the causal research hypothesis (ie, that a given intervention causes a particular benefit)'. ${ }^{34}$ In their own words, in testing efficacy, "[a]nalyses are conducted that attempt to answer the narrowest, "mechanistic" question'. 34 Conversely, 'effectiveness' is associated with the 'pragmatic' challenge of 'determin[ing] the effects of an intervention under usual conditions, with 'all the noise inherent therein'. ${ }^{34}$ Hence, they refer to the continuum as an explanatory-pragmatic one.

Inquiring into the ontological commitments that these distinctions uphold requires that we pose a simple, but often taken for granted, question: what must nature be made of such that, in order to provide an 'explanation' of a biomedical effect upon the body, one must look for it in conditions that allow one to simplify the empirical complexity of biomedical and bodily processes so that a single causal factor can be put to the test? The answer is that, while tangled up in empirically complexindeed, 'noisy'-circumstances (involving material infrastructures, economic conditions, cultural traditions, individual subjectivities, diverse biological contingencies, etc), the reality with which biomedicine would be concerned is deemed to be ontologically simple, ultimately made of single, atomic, entities and activities that may be accessed by means of controlled analysis. In other words, because bodies, as parts of nature, are deemed ontologically simple, in order to explain their functioning one must devise a methodology that allows one to cut through the apparent empirical, practical, social, economic, cultural and biological complexity that would otherwise impede an understanding of the reality of health conditions. If this position seems rather uncontroversial, it is because it has not just been at the heart of the RCT, or of EBM, but at the centre of modern scientific materialism since the seventeenth century. This is what philosopher Alfred North Whitehead termed the doctrine of 'simple location':

To say that a bit of matter has simple location means that, in expressing its spatio-temporal relations, it is adequate to state that it is where it is, in a definite finite region of space, and throughout a definite finite duration in time, apart from any essential reference of the relations of that bit of matter to other regions of space and to other durations of time. ${ }^{23}$

While EBM's hierarchies of evidence sustain that the statistical correlation based on the comparison of randomised groups provides, in and of itself, sufficient evidence that the treatment in question works both in the conditions in which it was tested and also that it should work in other conditions too, ${ }^{35} 36$ the problem with the doctrine of simple location, as Whitehead noted, ${ }^{23}$ is that it creates great difficulties for induction. For 'if in the location of configurations of matter throughout a stretch of time there is no inherent reference to any other times, past or future, or indeed, to any other configurations of matter in other regions of space, 'it immediately follows that nature within any period [or region] does not refer to nature at any other period [or region]'.23
For this reason, as the language of the epidemiological continuum described above reveals, many EBM proponents and analytical philosophers of medicine argue that the RCT in fact relies, or should rely, on an ontology of mechanisms that can provide a causal explanation for the correlations obtained. ${ }^{4} 3738$ Again, this mode of thought has a long history in medicine and biology. While it was Claude Bernard who famously claimed that ' $[w]$ e cure [a health condition] always without any exception, when we place ourselves in the known experimental conditions for reaching this goal, ${ }^{39}$ the mechanistic theory of living organisms can be traced all the way back to Descartes, and perhaps even to Aristotle. ${ }^{19}$ Equally, that the inductive logic of the method of difference requires the support of mechanistic assumptions was already anticipated by Mill himself, ${ }^{30}$ when he suggested that the 'ground' of all inductions must be the ontological premise that nature is made of a complex of uniform patterns. These he referred to as laws of nature.

Interestingly, by assuming that the realities of health and disease are mechanistic realities that hold with a significant degree of probability across space and time, this ontology of mechanisms is also said to contribute to the task of translating efficacy into effectiveness or of extrapolating the 'efficacy' findings of an RCT to other populations, 'because it adds precious knowledge about the similarities between the test and target populations'. ${ }^{4}$ However, if a mechanistic ontology can add knowledge to the problem of translating efficacy, it is also because it presupposes it. Indeed, because of the mechanistic ontology of nature that underpins EBM, measures of 'efficacy' are deemed evidential of the existence of hypothesised biological mechanisms of health and disease. Hence, the task of making such evidence effective outside the conditions of the trial is thus one of complementing it with one of a different order. That is, not other evidence of the nature of processes of health and disease, but evidence of something else entirely, namely of the complex, noisy, empirical, socio-economic, infrastructural, legal, cultural and biological situations in which biomedical mechanisms are meant to intervene.

We can thus begin to disclose the image of nature that underpins EBM conceptions of evidence. For the bifurcation of orders of evidence at the heart of mechanistic reasoning reveals a deeper bifurcation of the order of reality itself. According to the ontologic advanced by EBM, there would be, on the one hand, a mechanistic reality responsible for the nature and causes of health and disease, and on the other, a seemingly complex, noisy and contingent set of circumstances in which biomedical interventions must ultimately dwell, but which remain ontologically distinct from the mechanisms that underpin the nature of health and disease as such.

In this sense, the issue with a mechanistic ontology is not that it simply ignores the social, cultural and gendered biases of biomedical knowledge-production, ${ }^{40}$ or that the 'knowledge' it produces can never be disentangled from the way in which ethical, commercial and political priorities are set. ${ }^{41}$ Neither is it that it ignores the importance of human, social, cultural, economic and interpersonal dimensions of the experience of health and illness, ${ }^{42}$ or of clinical decision-making. ${ }^{43}$ It is all of this and more. The problem is also that by positing the explanation of biomedical interventions-and hence of the realities of health and disease-as a matter of responding to mechanistic questions, the distinction between 'efficacy' and 'effectiveness' on which EBM rests creates a bifurcation between two orders of reality. While one of them is conceived as essential to the question of what the nature of health is and what causes it-namely the mechanistic evidence that is either implicitly or explicitly 
provided by the testing of 'efficacy'-the other-the observational evidence of effectiveness-is conceived as accidental, proper to the empirical circumstances in which such causes may operate. In so doing, what is being reduced is not just the experience of being healthy or ill, but the very nature of health and disease. There is, in other words, a reduction of the question of what nature can do to the question of how biomedical science can explain it, namely through mechanisms. Meanwhile, the complex, varying and fundamentally situated realities of what in RCT parlance are termed 'real-world conditions' are not conceived as part of the same reality to which the nature of health and disease itself is said to belong.

The practical effect of reducing the question of what nature may be capable of to the very different question of what contemporary forms of biomedicine may be capable of saying about it is that, when the efficacy of an intervention is established, this experimental achievement tends, 'in policy discourse, [...] to trump all other considerations, instituting some degree of neglect toward the local and material relations that would need to be engaged to achieve the necessary outcomes or that might otherwise be affected by the intervention'. ${ }^{44}$ Similarly, in clinical practice, the experimental achievement of efficacy serves as a golden measure to establish processes of standardisation of care $^{33}$ while displacing clinical knowledge. ${ }^{45}$

Epistemologically, moreover, this bifurcation creates something of a zero sum game. For when the situated, empirical conditions of implementation of a treatment deemed 'efficacious' are met, this circumstantial evidence is seen to provide confirmatory support that the essential evidence of its efficacy is in fact real. Conversely, however, when the implementation of a treatment declared 'efficacious' fails, nothing about the nature of the process of health and disease can be learned from its effective failure because the failure to gain evidence for effectiveness can easily be ascribed, as it often is, ${ }^{46}$ to the unfavourable circumstances of its implementation (eg, poor compliance with treatment protocols, inadequate infrastructures, cultural prejudices against medical authority, etc). In other words, because measures of effectiveness are seen to provide evidence of an entirely different order than those of efficacy, either the situated achievement of 'real-world effects' conforms to the measures obtained under controlled experimental conditions and thus confirms the mechanistic 'nature' of health conditions that biomedicine already knew in advance or effectiveness becomes a practical, empirical 'obstacle' to be overcome, instead of a problem capable of eliciting qualitatively new questions and possibilities about the reality of the condition in question (M Savransky. The social and its problems: on problematic sociology. In: Marres et al. ed. Inventing the social. London: Mattering Press, forthcoming). Furthermore, from the point of view of the multidisciplinary division of labour that is involved in biomedical cultures, the consequences of this are, certainly, that once the RCT has done its job of determining the nature of the condition, it is the whole array of social scientists and humanities practitioners who become tasked with learning what it is about the circumstances of implementation that may pose the obstacle and how the latter may be removed (M Rosengarten. A peculiar demand: the social in HIV, $\mathrm{TB}$ and Ebola and the speculative possibilities of infection. In: Marres et al. ed. Inventing the social. London: Mattering Press, forthcoming).

\section{WHAT IS NATURE CAPABLE OF? HEALTH IN A WORLD OF CONNECTED SITUATIONS}

In light of the above discussion, it becomes clear that the call for a 'critical medical humanities' that can engage with questions of ontology in biomedical cultures, with questions, that is, not just of the contexts, meanings and circumstances in which health and disease 'happen', but of the heterogeneous processes by which the realities of health and disease are constituted and possibly transformed, is indeed critical. Because, if the diagnosis provided by Viney $e t a l^{1}$ is accurate, and we can understand the heterogeneous field of medical humanities, and of social studies of medicine, as swaying between the Scylla of 'a service or utilitarian model, which accommodates but does not actively seek to challenge pre-existing power structures and epistemological divisions of labour within biomedicine', and the Charybdis of 'a medical humanities imagined to be antagonistic, noisy, and opinionated', ${ }^{1}$ then what seems to be required are not new and stronger criticisms but an entirely different ethos of intervention in the realities of health. One that can find, in the long-standing resistance against positivist reductionisms, ${ }^{47}$ in the attention to the empirical complexities of medical practices, ${ }^{48}{ }^{49}$ in the sensitivity and practical engagements with narratives as well as patient-clinician relations, ${ }^{50-52}$ in their engagements with the arts in healthcare, ${ }^{53} 54$ as well as in all of the other habits and strategies by which the field of medical humanities is composed, crucial resources to intervene creatively and transform the ontological commitments on which the political, epistemological, practical and clinical consequences of biomedical research hinge.

What seems to be required, in other words, is an ethos that, in resisting clear-cut bifurcations between 'the essential' and 'the accidental', 'the biological' and 'the social', 'the natural' and 'the cultural', may become oriented towards the construction of new, inventive, plural and always partial descriptions and proposals concerned with the question of what the realities of health and disease are made of. Descriptions and proposals whose function is neither to denounce the practices of biomedical scientists when they are faced with the experimental event of an intervention that has 'worked' in controlled situations, nor simply to replace the mechanistic ontology of EBM for another, equally dogmatic, ontology. For as we have shown above, one cannot discern what counts, and what does not count, as 'evidence', unless one already possesses an answer to the question of what the world is made of. By the same token, one cannot provide 'evidence' that the world is in fact made in that way without falling into an infinite regress of evidences and assumptions all the way down. For this reason, questions of ontology are always speculative. Seeking to cultivate a speculative ethos, we approach such questions with the aim not of settling, once and for all, what the reality of health must be, but with the aim of experiencing the possible consequences, for humanistic and social scientific engagements with biomedical practices, situations and rationalities, of entertaining the sense of humility, curiosity and trust induced by propositions of a possible world that keeps open the question of what nature may be capable of. $^{55}$

In order to do this, in what follows we experiment with ontological questions by attempting to sketch out some elements of an image of the world where mechanisms can be regarded as part of the specific modes of explanation that stem from the practices of contemporary biomedical sciences, rather than as the very realities of health and disease to which every other form of evidence must conform. An image where practices of biomedical research do not lose their hold, but can witness a mutation of the manner in which they take hold. An image where RCTs are neither rendered futile nor allowed to occupy the position of the gold standard against which every other health practice may be judged, and to which they must be 
subjected. Contrary to what one might expect, the image we seek to propose is not one of a world of chaotic, discontinuous contingencies, but one of fragile, partial and dynamic connections between heterogeneous, situated and self-organising forms of order. Indeed, our proposition is that to think of health and disease as always situated achievements can contribute to cultivating existent and novel habits of the medical humanities towards the question of what nature may be capable of.

But what do we mean by a 'situation'? We must begin by expanding the scope of the notion of situation beyond the otherwise important concept of 'situated knowledges' that, ever since Donna Haraway proposed it in the late $1980 \mathrm{~s},{ }^{56}$ has resonated amply in most appeals to 'situatedness' in the social sciences and humanities. In Haraway's version, becoming situated constitutes a practice of 'embodied objectivity' that avows the marked, local and partial perspective of a knowing subject within the specific milieus that make scientific enterprises possible. Rather than drawing directly on this notion of situated knowing, our concept of a 'situation' is here inspired by the work of pragmatist and continental philosophers, ${ }^{18-25}$ as well as evolutionary biologists, ${ }^{26} 57$ for whom the appeal to the 'situated' is not epistemological but ontological-it is an image of a world-in-the-making, composed of plural, spatially and temporally interconnected environments with their own partial and dynamic patterns of order and disorder, endurance and emergence, continuity and change. It is a proposition that invites us to keep open the question of what nature is capable of because it confronts us with the idea that, rather than mechanistically governed, 'the universe in its persistent becoming is richer than all our dreamings'. ${ }^{26}$

To think of the realities of health and disease as situated achievements is to attempt to conceive of the very objects of medical inquiry and intervention as themselves already partaking in situations-simultaneously biological, social, economic, cultural and so on-which at once sustain them and subject them to their own tests of survival and transformation. Against the assumptions of RCTs, we seek to entertain the thought that 'there is never any such isolated singular object [...]; an object $[\ldots]$ is always a special part, phase, or aspect, of an environing experienced world- a situation'. ${ }^{26}$

Thus, while RCTs locate mechanisms through the abstraction of discrete, isolated entities and variables from their 'confounding' environments, the practice of situating, by contrast, does not allow such clear-cut distinctions. Rather, both an object and its situation are entangled, spatially and temporally, to one another such that both become co-determined through their specific, reciprocal transactions and exchanges. ${ }^{58}$ In this way, for example, an organ would constitute the situation of its composing cells, while simultaneously, an object situated in the body; the body constitutes an object with respect to its biological, social and cultural milieus, and these in turn constitute elements of a global, heterogeneous ecology. Thus, situations are without essence-that is, neither 'merely' social nor simply biologicaland the concrete identification of their limits is always a matter of empirical inquiry. ${ }^{19}$

The realities of health and disease can also be approached as situated events and processes that are defined not by stable mechanisms but by the dynamic relationships that cells sustain with organs, organs with bodies, and that bodies sustain with their environing physical, social, cultural, economic and ecological situations. Rather than be defined in terms of mechanisms to which an accidental environment may happen, to think of health and disease in a world of situations is to undo the bifurcation between what is essential and what is accidental.
It demands that one resists splitting off 'nature' from the noisy empirical conditions in which, by way of which, health and disease come to exist in specific ways. Put differently, it demands that one refrains from bifurcating nature-that one resists splitting off nature from itself-and that one includes in it not only forms of order and organisation of diverse scope, but also the myriad of contingent events and circumstances that also compose it. Thus, health ceases to be the result of underlying, stable mechanisms and becomes a function of the relationship between organisms and their internal and external environments. In this sense, the French historian and philosopher of medicine Georges Canguilhem, whose epistemological vitalism made him singularly attuned to the speculative question of what nature may be capable of, ${ }^{18} 59$ provides an adequate, if provocative, definition for health in a world of situated connections: 'Health', he proposes, 'is a margin of tolerance for the inconstancies of the environment'. ${ }^{20}$

To define the reality of health in this way is certainly to resist its reduction to a mechanistic ontology, but it is not for that reason to reject the potential value of mechanisms as useful abstractions that biomedical practices may construct to explore biological processes. Biomedicine, as we discuss below, may often provide successful and wide-ranging accounts of nature and the body, but no explanation can ever hope to provide a final response to the question of what nature can do. In other words, while the environment may be defined by the physical sciences in terms of mechanisms and probabilities, and while biomedicine may use those same terms to attempt to occasionally provide effective accounts of the realities of health and disease, we must resist the temptation to reduce the nature of medical realities to biomedical explanations. For, as Canguilhem argues, ${ }^{20}$ just as the operative state of a mechanism is not 'health' but order, and disorder is not evidence of a mechanism's 'disease', 'there is no health for a mechanism'. If health can be thought as a margin of tolerance for the inconsistencies of the environment, to be healthy is to be capable of responding to the surprises and unexpected changes that situations pose to the organisms that compose them. Rather than be premised on the order and stability of universal causal principles, then, in a world of connected situations health becomes a function of novelty. A responsive function, that is, to the novel, situated ways in which the question 'what is nature capable of?' is posed.

Entertaining situated, dynamic, medical ontologies, moreover, enables us to make sense of, and integrate, other forms of 'evidence' of the realities of health and disease produced by qualitative methods in the humanities and social sciences that put mechanistic assumptions about nature at risk. One interesting example of this is what medical anthropologist Margaret Lock has termed 'local biologies'. ${ }^{60} 61$ This term has been coined to account for the fact that differences in individual bodies, such as differences between periods and symptoms of the end of menstruation in North American and Japanese women, ${ }^{60} 62$ for instance, cannot be reduced to questions of cultural belief, but must be understood as 'local entanglements among historical and cultural activities, technoscientific interventions, and the biology of individual ageing'. ${ }^{63}$ In contrast with the image of a mechanistic nature, such studies point instead to the dynamic processes of differentiation, at once biological, historical, social and technological, by which, sometimes and in some places, 'continual interactions of biological and social processes across time and space [...] eventually sediment into local biologies' endowing bodies with different biological compositions and functions. $^{63}$ 
Does this mean that in this alternative image of nature, where realities of health are always situated, the generalising aspirations of biomedical claims to the 'efficacy' of treatments become, by definition, simply spurious claims? The answer to this question, we suggest, is 'no'. For to think in terms of situations does not lead to the image of an entirely indeterminate nature where enduring and wide-ranging patterns of order, and thus, of causation, would be impossible. Situations are not another name for solipsistic, isolated systems that bear no connection with each other. Rather, just as they are internally connected to the elements that compose them, there are no ontological gaps between one situation and another. In other words, situations are always connected to each other, allowing for the possibility of wide-ranging patterns of order to obtain, such that the achievement of a causal pattern accomplished within the situation that the methodological rules of the RCT themselves institute may, sometimes, be stable enough to witness the same effects in situations otherwise differently composed.

However, whereas a mechanistic ontology assumes that the world is made of such general patterns, in this alternative image the connections between situations are 'typically loose, incomplete, and themselves susceptible to potential change'. ${ }^{25}$ In this way, a claim to efficacy provides no guarantee, in and of itself, that such generalisations will hold. Every taking hold is the achievement of establishing an effective connection between a biomedical intervention and the environment it has sought to affect. Because of this, however, to conceive of nature in terms of a system of multilayered, connected and dynamic situations does force us to come to terms with the fact that one cannot shift the responsibility of the failure of a declared 'efficacious' drug to be 'effective' to the noisy empirical situations in which bodies live, change, become sick or health, and die. ${ }^{44}$ Because just as 'anything that can exist at any place and time occurs subject to tests imposed upon it by surroundings', ${ }^{21}$ so should biomedical innovations be put to the test of the environments in which they seek to intervene. And such a test requires a coming to terms with both the determinate and indeterminate dimensions of concrete situations.

In this way, the inferences that the method of the RCT relies upon may be justified only to the extent that the forms of order on which such inferences are grounded do empirically recur in the novel situation in which they are deployed. But in a nonmechanistic world, no claim to evidence of efficacy can authorise biomedical explanations to judge a situation on the basis of whether or not the latter conforms to it. By the same token, neither can 'efficacy' become a guarantee, however probable or plausible within an experimental population, that such 'evidence' will hold across other bodies, spaces and times. By contrast, the failure of a biomedical intervention to become 'effective', to resituate a claim to efficacy, becomes an opportunity to learn something new about the production of the realities of health and disease. It becomes an opportunity to ask again the question of what nature is capable of.

\section{CONCLUSION: MEDICAL HUMANITIES AND SPECULATIVE DREAMING}

Expanding on the recent call for medical humanities to intervene in questions of the ontology of health, in this paper we have sought to offer a speculative orientation to developing such interventions in relation to some of the ontological commitments on which contemporary biomedical cultures rest. We have suggested that crucial to this task is an approach to ontology that treats it not as a question of first principles, but as a matter of the consequences of the images of nature that contemporary biomedical research practices espouse when they make claims to evidence, as well as the possible consequences of imagining different worlds in which health and disease processes partake. We have thus sought to disclose the way in which the mechanistic ontology that evidence-based biomedical research methodologies uphold simultaneously grants them a special epistemic status and authorises a series of problematic consequences for understanding and intervening practically in the concrete realities of health. As a response, we have attempted to briefly sketch an alternative ontological proposition that, without disqualifying RCT-based evidence, may enable practices in the medical humanities, in the social sciences, in the arts, and so on, to collaboratively resist the reduction of the realities of health and disease to biomedicine's current forms of explanation and venture into situated forms of learning, understanding and intervening.

Such an alternative ontology of situated and interconnected processes of health and disease is, however, nothing but a proposition. Just like the ontological assumptions on which EBM rests, ours too is, in the full sense of the term, a fiction. Unlike those that underpin EBM, however, our fiction is not aimed at creating a new consensus over what the reality of health really is, but to lure scholars in the medical humanities and allied fields to engage-in their own ways, with their own means and in relation to always specific situations-with speculative questions of the ontological processes of production, constitution and transformation of health, disease and illness.

Unlike forms of antagonistic critique, ${ }^{15}$ speculative thought does not operate by attempting to awaken those it addresses to a reality from which they would have been blinkered. It is not designed to shock, to make those it addresses-either biomedical scientists or medical humanities scholars-lose their hold and shatter their certainties. To speculate, by contrast, is to respect the importance of what gives others a hold. As Stengers puts it, ${ }^{44}$ a speculative intervention operates by posing the question 'What is required by your hold?'. Such a question 'affirms and presupposes that the others' dreams, like yours, are created according to the means of their own adventure, and to this extent this question constitutes a test. ${ }^{, 44}$ It is a test in the sense that to ask what is required by the hold of contemporary biomedical forms of evidence is a question that resists the obviousness with which such forms of evidence have been endowed. Rather than seek solutions to a problem that is presented as given, as seems to be the case in many debates around so-called implementation sciences, ${ }^{64}{ }^{65}$ we have sought to speculate on the possibility of transforming the image of nature in which the problem is posed. Thus, we have imaginatively explored the ways in which an image of nature that resists clear-cut bifurcations between 'the essential' and 'the accidental', 'the natural' and 'the cultural', and 'the biological' and 'the social' may enable problems to be resituated and new possibilities to become available. As such, speculation poses a challenge not to those who trust conventional forms of biomedical evidence, but to those who are invested in the power that comes with their acquired obviousness.

Yet, to ask 'what is required by your hold?' is also a question that 'one dreamer can address to another dreamer', ${ }^{44}$ with the hope that one dream may lure the dream of the other into new directions or may evoke the possibility of a new kind of collaborative dreaming. Rather than simply denouncing the mechanistic ontologic of 'evidence-based' biomedical research methodologies, intervening speculatively in ontological questions involves creatively deploying the various habits of the medical humanities, as well as those of other scientific and artistic practices with which it 
may and often does collaborate, to become sensitive to the question-rather than the answer-of what nature may be capable of. It is a speculative proposition addressed to another dreamer, in recognition that 'the universe in its persistent becoming is richer than all our dreamings'. ${ }^{19}$ This does not mean shying away from creating new fictions or from communicating other dreams that would differently characterise the natures of health and disease. It invites other dreamers to engage in dreaming but not simply as an exercise in unconstrained imagination as if speculative questions would fall off a sky of ideas. Speculative dreaming, by contrast, is always partial, plural and practical, characterised by the risks it takes when it thinks alongside other situations, practices and experiences that prevailing regimes of evidence would deem improbable, artifactual or confounding. ${ }^{58}$ The risks of speculative dreaming, ultimately, are ones that subject dreaming to the test of its own consequences upon the dreams of others.

Twitter Follow Martin Savransky at @msavransky

Funding British Academy ('Towards a Concept of Situated Efficacy' Small Grant).

Competing interests None declared.

Provenance and peer review Not commissioned; externally peer reviewed.

\section{REFERENCES}

1 Viney W, Callard F, Woods A. Critical medical humanities: embracing entanglement, taking risks. Med Humanit 2015;41:2-7.

2 Howick J. The philosophy of evidence-based medicine. Oxford, UK: Wiley-Blackwell, 2011.

3 Cartwright N, Hardie J. Evidence-based policy: a practical guide to doing it better. Oxford, UK: Oxford University Press, 2011.

4 Clarke B, Gillies D, Illari $P$, et al. The evidence that evidence-based medicine omits. Prev Med 2013;57:745-7.

5 Deleuze G. Difference and Repetition. New York: Columbia University Press, 1994.

6 Greco M. On the art of life: a vitalist reading of medical humanities. Sociol Rev 2008:56:23-45.

7 Simon JR. Advertisement for the ontology for medicine. Theor Med Bioeth 2010;31:333-46.

8 Stengers I. Cosmopolitics I. Minneapolis, MN: Minnesota University Press, 2010.

9 Bensaude-Vincent B, Stengers I. A History of Chemistry. Cambridge, MA: Harvard University Press, 1996

10 Nathan T, Stengers I. Médecins et sorcieres. Paris: Les Empêcheurs de Penser en Rond, 1995.

11 Chertok L, Stengers I. A Critique of Psychoanalytic Reason: Hypnosis as a Scientific Problem from Lavoisier to Lacan. Stanford, CA: Stanford University Press, 1992.

12 Stengers I. Cosmopolitics II. Minneapolis, MN: University of Minnesota Press, 2011.

13 Pignarre P, Stengers I. Capitalist Sorcery: Breaking the Spell. London: Palgrave Macmillan, 2011.

14 Stengers I. The Invention of Modern Science. Minneapolis, MN: University of Minnesota Press, 2000.

15 Goffey A. Homo Immunologicus: on the limits of critique. Med Humanit 2015;41:8-13.

16 Whitehead AN. The Function of Reason. Boston, MA: Beacon Press, 1958.

17 Grossman J, Mackenzie FJ. The Randomized Controlled Trial: gold standard, or merely standard? Perspect Biol Med 2005;48:516-34.

18 Canguilhem G. The Normal and The Pathological. Brooklyn, NY: Zone Books, 1991.

19 Canguilhem G, Knowledge of life. Fordham, CA: Fordham University Press, 2008.

20 Canguilhem G. Writings on medicine. Fordham, CA: Fordham University Press, 2012.

21 Dewey J. Logic: the theory of inquiry. New York, NY: Irvington Publishers, 1938.

22 Dewey J. Experience and Nature. Mineola, NY: Dover Publications, 1958.

23 Whitehead AN. Science and the modern world. New York, NY: Free Press, 1967.

24 James W. Pragmatism and the meaning of truth. Seaside, OR: Watchmaker Publishing, 2011.

25 Connolly W. A world of becoming. Durham, NC: Duke University Press, 2011.

26 Kaufmann S. Investigations. Oxford, UK: Oxford University Press, 2000.

27 Sacket DL, Rosenberg WM, Gray JA, et al. Evidence based medicine: what it is and what it isn't. BMJ 1996;312:71-2.

28 Rothwell PM. External validity of randomised controlled trials: "to whom do the results of this trial apply?". Lancet 2005;365:82-93.
29 Timmermans S, Berg M. The Gold Standard: the challenge of evidence-based medicine and standardization in health care. Philadelphia, PA: Temple University Press, 2003.

30 Mill JS. A system of logic. New York, NY: Harper and Brothers Publishers, 1882.

31 Wills C, Moreira T. eds. Medical proofs, social experiments: clinical trials in shifting contexts. Farnham, UK: Ashgate Publishers, 2010.

32 Wills CM. The alchemy of clinical trials. BioSocieties 2007;2:85-99.

33 Timmermans S, Epstein S. A world of standards but not a standard world: towards a sociology of standards and standardization. Ann Rev Sociol 2010;36: 69-89.

34 Thorpe KE, Zwarenstein M, Oxman AD. et al A pragmatic-explanatory continuum indicator summary (PRECIS): a tool to help trial designers. J Clin Epidemiol 2009;62:464-75.

35 Cartwright N. What is this thing called efficacy? In: Mantzavinos C. ed. Philosophy of the social sciences: philosophical theory and scientific practice. Cambridge, UK: Cambridge University Press, 2009:185-206.

36 Cartwright N. Are RCTs the Gold Standard? BioSocieties 2007;2:11-20.

37 Russo F, Williamson J. Interpreting causality in the health sciences. Int Stud Philos Sci 2007;21:157-70.

38 Solomon M. Just a paradigm: evidence-based medicine in epistemological context. Eur J Philos Sci 2011;1:451-66.

39 Bernard C. An introduction to the study of experimental medicine. Mineola, NY: Dover Publications.

40 Goldenberg MJ. On evidence and evidence-based medicine: lessons from the philosophy of science. Soc Sci Med 2006;62:2621-32.

41 Abraham J. Drug trials and evidence bases in international regulatory context. BioSocieties 2007;2:41-56.

42 Garden R. Who speaks for whom? Health humanities and the ethics of representation. Med Humanit 2015;41:77-80.

43 Saunders J. The practice of clinical medicine as an art and as a science. Med Humanit 2000;26:18-22.

44 Race K. Framing responsibility: HIV, biomedical prevention, and the performativity of the law. J Bioeth Inq 2012;9:327-38.

45 Jensen UJ. The struggle for clinical authority: shifting ontologies and the politics of evidence. BioSocieties 2007;2:101-14.

46 Glasgow R, Lichtenstein E, Marcus AC. Why don't we see more translation of health promotion research to practice? Rethinking the efficacy-to-effectiveness transition. Am J Public Health 2003;93:1261-7.

47 Evans M, Finlay IG. eds. Medical humanities. London: BMJ, 2001.

48 Berg M, Mol A. eds. Differences in Medicine: Unraveling practices, techniques, and bodies. Durham, NC: Duke University Press, 1998.

49 Michael M, Rosengarten M. Innovation and biomedicine: ethics, evidence and expectation in HIV. Basingstoke, UK: Palgrave Macmillan, 2013.

50 Hurwitz B, Greenhalgh T, Skultans V. eds. Narrative research in health and illness. Malden, MA: BMJ Books, 2004

51 Woods A. The limits of narrative: provocations for the medical humanities. Med Humanit 2011;37:73-8.

52 Atkinson S, Macnaughton J, Saunders C, et al. Cool intimacies of care for contemporary clinical practice. Lancet 2010;376:1732-3.

53 Longden E, Davis P, Billington J. et a/ Shared Reading: assessing the intrinsic value of a literature-based health intervention. Med Humanit 2015;41:113-20.

54 Bates V, Bleakley A, Goodman S. eds. Medicine, health and the arts: approaches to the medical humanities. Abingdon: Routledge, 2014.

55 Stengers I. Thinking with whitehead: a free and wild creation of concepts. Cambridge, MA: Harvard University Press, 2011.

56 Haraway D. Simians, cyborgs and women: the reinvention of nature. London, UK: Free Association Books, 1991

57 Margulis L, Sagan D. What is life? Berkeley, CA: University of California Press.

58 Savransky M. The adventure of relevance: an ethics of social inquiry. Basingstoke, UK: Palgrave Macmillan, 2016.

59 Greco M. On the vitality of vitalism. Theory Cult Soc 2005;22:15-27.

60 Lock M. Encounters with ageing: mythologies of menopause in Japan and North America. Berkeley, CA: University of California Press, 1993.

61 Lock M. The epigenome and nature/nurture reunification: a challenge for anthropology. Med Anthropol 2013;32:291-308.

62 Melby MK, Lampi M. Menopause: a biocultural perspective. Ann Rev Anthropol 2011;40:53-70.

63 Lock M, Nguyen V. An Anthropology of Biomedicine. Oxford, UK: Wiley-Blackwell, 2013.

64 Barker PM, Reid A, Schall MW. A framework for scaling up health interventions: lessons from large-scale improvement initiatives in Africa. Implement $\mathrm{SCi}$ 2016:11:12.

65 Sobo EJ, Bowman C, Gifford AL. Behind the scenes in health care improvement: the complex structures and emergent strategies of Implementation Science. Soc Sci Med 2008:67:1530-40. 